\title{
Programmable CRISPR interference for gene silencing using Cas13a in mosquitoes
}

Aditi Kulkarni, Wanqin Yu, Alex Moon, Ashmita Pandey, Kathryn A. Hanley, Jiannong Xu*

Department of Biology, New Mexico State University, PO Box 30001 MSC 3AF, Las Cruces NM, 88003, USA

Aditi Kulkarni, aditik@nmsu.edu, (575) 646-5936

Wanqin Yu, ivyyu@nmsu.edu, (575) 646-5936

Alex S. Moon, alexmoon@nmsu.edu, (575) 646-5936

Ashmita Pandey, ashmita@nmsu.edu, (575) 646-5936

Kathryn A. Hanley, khanley@nmsu.edu, (575) 646-4583

Jiannong Xu, jxu@nmsu.edu, (575) 646-7713

Running title: Cas13a-CRISPRi for gene silencing in mosquitoes

*Corresponding: Jiannong Xu, jxu@nmsu.edu, (575) 646-7713, Department of Biology, New Mexico State University, PO Box 30001 MSC 3AF, Las Cruces NM, 88003, USA 


\begin{abstract}
In the CRISPR-Cas systems, Cas13a is an RNA-guided RNA nuclease specifically targeting single strand RNA. We developed a Cas13a mediated CRISPR interference tool to target mRNA for gene silencing in mosquitoes. The machinery was tested in two mosquito species. A Cas $13 a$ expressing plasmid was delivered to mosquitoes by intrathoracic injection, and Cas 13 a transcripts were detectable at least10 days post-delivery. In Anopheles gambiae, vitellogenin gene was silenced by $V g$-crRNA injection two hours post-blood meal, which was accompanied by a significant reduction in egg production. In Aedes aegypti, the $\alpha$ - and $\delta$-subunits of COPI genes were silenced by a post-blood meal crRNA injection, which resulted in mortality and fragile midguts, reproducing a phenotype reported previously. Co-silencing genes simultaneously is achievable when a cocktail of target crRNAs is given. No detectable collateral cleavages of nontarget transcripts were observed in the study. This study adds a programmable CRISPR tool to manipulate RNA in mosquitoes.
\end{abstract}

Keywords CRISPR-Cas13a, RNA interference, Anopheles gambiae, Aedes aegypti, gene silencing, CRISPRi 


\section{Introduction}

Characterization of mosquito life traits via functional genomics approaches can inform innovative control strategies through the identification of genes involved in development, hostseeking, blood feeding, digestion, fecundity, immunity, xenobiotic metabolism and insecticide resistance. The last is perhaps the most immediately impactful, as, with increasing insecticide resistance, the array of options for vector control is shrinking and in dire need of replenishment. RNA interference (RNAi) based approaches have been widely used to identify genes that are relevant to vector competence, and RNAi-based effectors for mosquito control have been developed ${ }^{1-5}$. As an alternative or complement to RNAi-based tools, CRISPR-Cas9 based genome editing tools have been developed in the mosquito research field ${ }^{6-8}$.

CRISPR-Cas systems are adaptive immune mechanisms used by prokaryotes to defend against invading DNA and RNA ${ }^{9-12}$. Cas9 is a RNA-guided DNA nuclease and once assembled with a CRISPR guide RNA (sgRNA), Cas9 is able to cleave target DNA in a highly specific fashion, and DNA-targeting Cas9 has been harnessed for genome editing ${ }^{13,14}$. More recently, CRISPR-Cas9 based genome editing tools have been developed in the mosquito research field ${ }^{6-8}$. Furthermore, catalytically inactive Cas9 (dCas9) was adapted for manipulation of gene expression. The dCas9 can be fused with a gene repressor or transcription activator. Guided by CRISPR RNA, such dCas9 proteins are able to bind target promoter or exonic DNA sequence without cleavage, and either repress transcription (CRISPR interference, CRISPRi) or activate transcription of target genes (CRISPR activation, CRISPRa) ${ }^{15-17}$. Lately, Cas13 RNA nucleases 10, 18, 19, the new members in the CRISPR nuclease family, have been repurposed to specifically target endogenous RNAs as well as viral RNAs ${ }^{11,20-23}$. Most Cas 13 proteins are single "effector" proteins with two Higher Eukaryotes and Prokaryotes Nucleotide-binding (HEPN) domains ${ }^{10,24}$. Once loaded with a target-specific crRNA, a Cas13 protein will locate target RNA and execute nuclease activity to degrade the target. Unlike Cas9, no Protospacer Adjacent Motif (PAM) sequences are required for Cas13 to function. Although a Protospacer Flanking Site (PFS), A, U, C, may be present for PspCas13b activity ${ }^{25}$, no PFS is needed for $L w a C a s 13 a^{11}$. The Cas13s tested for human RNA knockdown thus far have demonstrated high specificity and exhibited negligible, and significantly fewer, off-target effects than matched RNAi short hairpin RNAs (shRNA) using to trigger RNAi 11, 25. In bacteria, Cas13 HEPN-nuclease is able to cleave not only the target-RNA in cis but also 
other non-target RNA present in trans ${ }^{10}$. Interestingly, no collateral effect was observed in three studies of CRISPR-Cas13 using human or plant cell lines ${ }^{11,25,26}$, but Cas13a associated collateral RNA cleavage was reported in human glioma cancer cells ${ }^{27}$. Notably, Cas13 can effectively silence several transcripts in parallel 11, 20, 26, 28. Taken together, CRISPR-Cas13 systems have become a new, exciting engine for CRISPRi ${ }^{29}$.

In this study, we engineered a construct to express Cas13a from Leptotrichia wadei in mosquitoes and demonstrated its efficacy in silencing genes in two mosquito species, Anopheles gambiae and Aedes aegypti, that are major vectors of malaria and mosquito-borne viruses, respectively.

\section{Materials and Methods}

\section{Plasmid construction}

The Cas13a from L. wadei belongs to the class 2 type VI RNA-guided RNA nucleases ${ }^{11}$. It's RNA targeting effect has been demonstrated in human and plant cells ${ }^{11,20,27,30}$. Plasmid pAcsgRNA-Cas9 was used as a template to engineer construct pAc-Cas13a (Fig. 1). Plasmid pAcsgRNA-Cas9 was a gift from Ji-Long Liu (Addgene plasmid \# 49330). The codons of LwaCas13a gene were optimized to Drosophila preference. The codon optimized Cas13a sequence was synthesized and cloned at GenScript (https://www.genscript.com). The Cas 9 coding sequence in plasmid pAc-sgRNA-Cas9 as replaced by the Cas 13 a coding sequence to create the pAc-Cas13a construct. The Cas13a sequence was confirmed by sequencing. The Cas $13 a$ was under the control of Drosophila actin (Ac5) promoter for constitutive Cas13a expression. The ampicillin resistance

Fig. 1. Cas13a construct and function. (A) Map of pAc-LwaCas13a (partial). (B)
Mechanism of Cas13a/crRNA mediated target RNA cleavage.

gene $(A m p R)$ allows selection of positive clones (Fig.1A). The plasmid was replicated in E.coli, and extracted using a QIAprep Spin Miniprep kit (Cat No.27104, Qiagen). The sequence of 
plasmid pAc-LwaCas13a was deposited in NCBI under accession number (in the process, to be provided).

Synthesis of crRNA

A crRNA consists of a 36-nt direct repeat (DR) sequence and 28-nt target RNA specific sequence $\left(\mathrm{N}_{28}\right)$, and the sequence of $\mathrm{N}_{28}$ is complementary to the target RNA sequence (Fig.1 B). The T7 promoter sequence (AGTTAATACGACTCACTATAGG) was added to the 5' end of the DR sequence (GATTTAGACTACCCCAAAAACGAAGGGGACTAAAAC) to enable crRNA synthesis using T7 RNA polymerase in vitro. The target specific sequences $\left(\mathrm{N}_{28}\right)$ used in this study are shown in Table 1. The selection of target $\left(\mathrm{N}_{28}\right)$ is straightforward as LwaCas13a does not require PFS for activity ${ }^{11}$. Please note, the target sequence for COPI- $\alpha$ was accidently designed as a-27nt sequence. For each $V g, C O P I-\alpha$ and $C O P I-\delta$, one target crRNA was used silencing, and for Caspar and Cactus, two target crRNAs were used. Template DNA duplexes of the crRNAs (T7-DR-N28) were synthesized at IDT Inc. (https://www.idtdna.com). The crRNAs were synthesized using T7-RNA polymerase (RPOLT7-RO ROCHE, Sigma-Aldrich). The crRNA synthesis reactions were set up in $40 \mu$ containing template DNA duplex $(1 \mu \mathrm{g}), 1 \mathrm{mM}$ each of nucleotides ATP, GTP, CTP and UTP, 10X reaction buffer, T7 RNA polymerase 40U, and RNase inhibitor $20 \mathrm{U}$. The reactions were incubated overnight at $37^{\circ} \mathrm{C}$ and stopped by heating the mixture at $65^{\circ} \mathrm{C}$ for 5 minutes. The crRNAs were treated with Turbo DNase I Kit (AM1907, ThermoFisher)

Table 1. The crRNA $\left(\mathbf{N}_{28}\right)$ sequences

\begin{tabular}{lll}
\hline Gene & $\begin{array}{l}\text { GenBank } \\
\text { accession }\end{array}$ & crRNA sequence $\left(\mathbf{N}_{\mathbf{2 8}}\right)$ \\
\hline$V g$ & XM_313104 & CACCTGCACCTTCACGCTGTCACCAGCC \\
COPI- $\delta$ & XM_001663354 & TGATAGACATACCGCACGGAATCTGTCT \\
COPI- $\alpha$ & XM_001663259 & ACCGCCTCTGCTTGTAGTTCCACACCT \\
Cactus-1 & XM_001650217 & ATCACCGTCGTCGTTCTGGTGGAAGTAC \\
Cactus-2 & XM_001650217 & TGATGCACAGGTCGTCCACCTTCATCGG \\
Caspar-1 & XM_021842247 & TCAACGCCGGACTCGGCCAGTGTCGTAC \\
Caspar-2 & XM_021842247 & TACCACTGCCACCGGCGGACGATCTCTG \\
Control & N/A & GACGCACATTCATAGTCTTCATCTGAGT \\
\hline
\end{tabular}


to remove template DNA. The crRNA yield was quantified using a NanoDrop and stored at -20 ${ }^{\circ} \mathrm{C}$ until use. Control crRNA (ctr crRNA) consisted of a randomly scrambled $\mathrm{N}_{28}$ nucleotide sequence, which had no homologous hit in the genomes of An. gambiae and Ae. aegypti.

Construct and crRNA Delivery

An. gambiae G3 strain and Ae. aegypti Puerto Rico strain were obtained from MR4 BEI and maintained using rearing conditions described previously ${ }^{31,32}$. The pAc-Cas13a construct $(0.5 \mu \mathrm{g} / \mu \mathrm{l})$ was delivered into one-day old adult female mosquitoes by intrathoracic injection. To aid construct delivery into cells, the plasmid was mixed with a transfecting agent FuGENE HD (E2311, Promega) at concentration of $1.6 \mu \mathrm{l}$ of FuGENE reagent with $10 \mu \mathrm{g}$ construct DNA in 20 $\mu \mathrm{l}$. The final concentration of construct DNA was $0.5 \mu \mathrm{g} / \mu \mathrm{l}$ in the mixture. Approximately, each An. gambiae mosquito received $100 \mathrm{nl}$ mixture, and each Ae. aegypti mosquito received $150 \mathrm{nl}$ mixture. Gene specific crRNAs were either delivered with the construct or separately at a later time point. For blood inducible genes, $V g$ and $C O P I$, female mosquitoes were given a blood meal three days post construct injection. Corresponding crRNAs $(0.5 \mu \mathrm{g} / \mu \mathrm{l}$, prepared in FuGENE as described above) were intrathoracically injected into mosquito hemocoel at two hours post blood meal.

\section{RNA isolation, cDNA synthesis and PCR}

Total RNA from whole mosquitoes was isolated using Trizol (Invitrogen) following the manufacturer's instruction. The RNA was treated with Turbo DNase I Kit to remove genomic DNA contamination, and then $1 \mu \mathrm{g}$ RNA was converted to cDNA using Protoscript II RT (M0368S, New England Biolabs) following the manufacturer's instruction. The PCR assays were performed using 1 1 1:5 diluted cDNA as template, $0.2 \mu \mathrm{M}$ primers (primer sequences are presented in Table S1) and $2 \times$ PCR Master mix (M0482S, NEB), with the following cycling parameters: 35 cycles of denaturing at $95^{\circ} \mathrm{C}$ for 15 seconds, annealing at a temperature optimal for the amplicon (Table $\mathrm{S} 1$ ) for 15 seconds, and extension at $68^{\circ} \mathrm{C}$ for 20 seconds with an extra $5 \mathrm{~min}$ in the last cycle for final extension.

\section{Statistical analysis}

In the $V g$ gene knockdown experiment, eggs were dissected from ovaries at day 3 post blood meal. The egg counts were compared between the $V g$ crRNA and control crRNA cohorts. 
The non-parametric Mann-Whitney test was used for statistical comparison of the egg numbers. In the COPI gene knockdown experiment, a survival curve was plotted using GraphPad Prism, and a Mantel-Cox analysis was performed to compare the survival between the COPI crRNA and control crRNA cohorts.

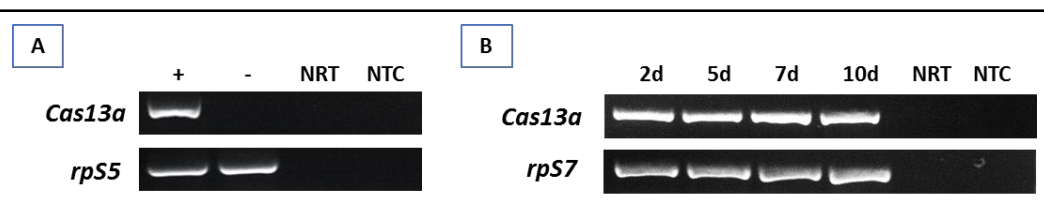

Fig. 2. Detection of Cas13a transcript in pAC-Cas13 injected mosquitoes. (A) RT-PCR of Cas13a in An. gambiae mosquitoes that received the construct (+) vs. control (-) at day 3 post plasmid delivery. NRT: No RT control; NTC: no template control. The rpS5 was used as a loading control. (B) Expression pattern of Cas13a transcript over a period of 10 days in Ae. aegypti, the $r p S 7$ was used as a loading control.

\section{Results}

\section{Cas 13a expression in mosquitoes post intrathoracic delivery}

A construct was engineered to express Cas $13 a$ gene by modifying the plasmid pAc-sgRNACas9, which was successfully used to transfect Drosophila cells for targeted genetic mutagenesis previously by Bassett et al. (2014) ${ }^{33}$. As shown in Fig. 1, Cas13a coding sequence is under the control of constitutive promoter Ac5. The plasmid was prepared with transfection reagent FuGENE HD and injected into thorax of one-day old mosquitoes. Cas $13 a$ transcription in whole mosquitoes was determined by RT-PCR. In An. gambiae, the RNA was sampled at day three post construct delivery, and Cas13a transcripts were detected (Fig. 2A). No Cas13a amplification was observed in the controls that did not receive the construct. In Ae. aegypti, the RNA samples were collected on day 2, 5, 7 and 10 post construct delivery, and Cas $13 a$ transcripts were detected in all of these time points (Fig. 2B).

\section{Cas13a mediated Vitellogenin gene silencing in An. gambiae}

In mosquitoes, yolk protein precursor vitellogenins $(\mathrm{Vg})$ are required for the vitellogenic stage in oogenesis after blood feeding ${ }^{34}$. To test Cas13a/crRNA mediated $V g$ silencing, the Cas13a construct was delivered to one-day old An. gambiae $(\mathrm{N}=120)$. Three days later, the mosquitoes were given a blood meal to induce $V g$ expression $(\mathrm{N}=96)$. To activate $V g$ knockdown, $V g$ crRNA 
$(\mathrm{N}=41)$ or control crRNA $(\mathrm{N}=40)$ were injected into the blood engorged females at $2 \mathrm{hr}$ post feeding. The $V g$ RT-PCR was used for verification of $V g$ knockdown. As shown in Fig. 3, the abundance of $V g$ transcript was reduced in females that received $V g$-crRNA as compared to females that received control-crRNA. As expected, successful $V g$ knockdown resulted in reduction in egg production (Fig. 3). The $V g$-crRNA treated mosquitoes produced on average $39 \pm 25$ (mean $\pm \mathrm{SD})$ eggs/female $(\mathrm{N}=33)$, while control mosquitoes produced $64 \pm 23$ eggs/female $(\mathrm{N}=32)$. The difference was statistically significant (Mann-Whitney test, $\mathrm{P}<0.001$ ). A second experimental replicate generated data with similar pattern showing significant reduction in egg numbers (MannWhitney test, $\mathrm{P}<0.01$, Fig. S1). The $\mathrm{Vg}$ proteins are produced by fat body and secreted into hemolymph, and subsequently deposited in developing oocytes via receptor-mediated endocytosis. The results suggest that the Cas 13 construct was successfully delivered into fat body cells, where the Cas $13 a$ was transcribed and translated into functional Cas13a proteins. Post blood feeding, $V g$ crRNAs were delivered into cells, where they were assembled with Cas13a to form a target specific RNA nuclease complex, which cleaved the $V g$ mRNA effectively.

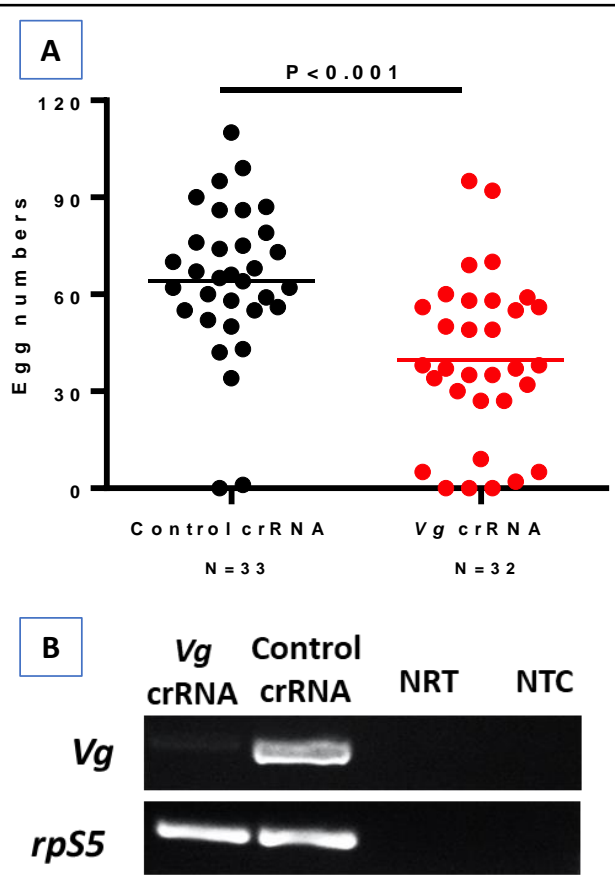

Fig. 3. An. gambiae $\mathbf{V g}$ knockdown reduced egg production. (A) Mosquitoes treated with Cas13a/Vg-crRNA had lower egg counts as compared to the cohort treated with Cas13a/ctrcrRNA (P<0.001). (B) Reduction of $V g$ transcripts was confirmed by RT-PCR. NRT: No RT control; NTC: no template control. The rpS5 was used as a loading control. 


\section{Cas13a mediated COPI gene silencing in Aedes aegypti}

The coatomer complex I (COPI) proteins are involved in blood digestion in Ae. aegypti ${ }^{35}$. The COPI complex consists of $\alpha, \beta, \beta^{\prime}, \gamma, \delta, \varepsilon$ and $\zeta$ subunits, each encoded by separate genes. The production of COPI proteins is blood inducible between 18-36 hr post blood meal in fat body and 24-48 $\mathrm{hr}$ post blood meal in ovaries ${ }^{35}$. As shown by other investigators, dsRNA-mediated knockdown of the genes encoding all but the $\varepsilon$ subunit led to blood meal-induced mortality ${ }^{35}$. Therefore, we targeted the COPI genes to test the gene silencing efficacy of the Cas 13a machinery in Ae. aegypti. The Cas13a construct was injected into one-day old mosquitoes $(\mathrm{N}=90)$. At day 3 post construct delivery, the mosquitoes were given a blood meal. The engorged mosquitoes $(\mathrm{N}=$ 75) were split into two cohorts, one was injected with the crRNAs specific for $\alpha$ and $\delta C O P I$ genes at $2 \mathrm{hr}$ hours post blood meal $(\mathrm{N}=27)$. The other cohort $(\mathrm{N}=29)$ was injected with the control crRNA. A subset of mosquitoes $(\mathrm{N}=5)$ in each cohort was sampled for knockdown validation by RT-PCR at $20 \mathrm{hr}$ post blood meal. Reduced abundance of the COPI transcripts was observed in

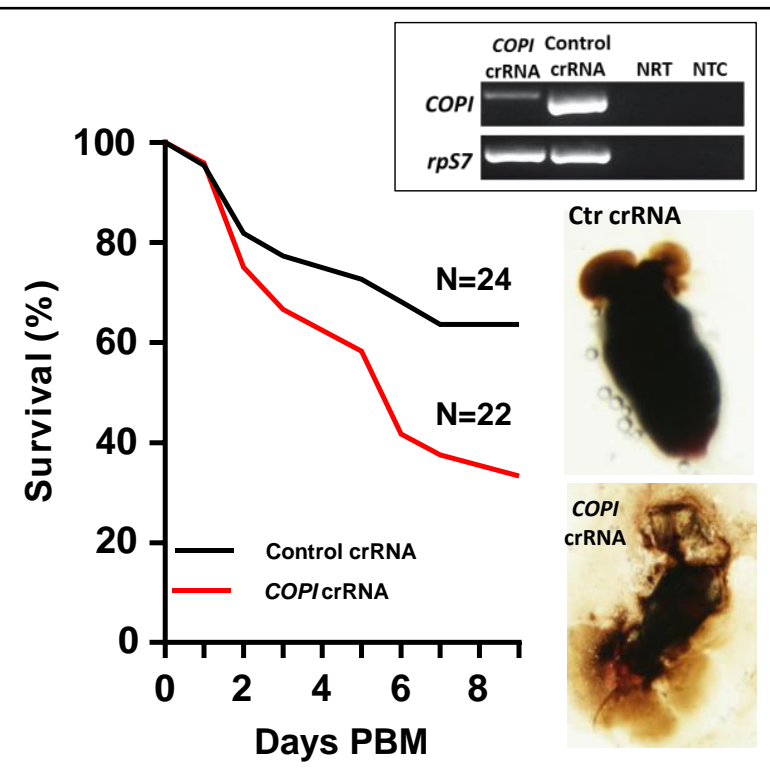

Fig. 4. Phenotypes of Ae. aegypti COPI knockdown. Cas13a/COPI-crRNA resulted in mortality post blood meal (PBM) and fragile midgut. Reduction of COPI transcripts was confirmed by RT-PCR (insert panel). NRT: No RT control; NTC: no template control. The rpS7 was used as a loading control.

the knockdown group (Fig. 4). The survival curves over 9 days post blood meal revealed a significantly lower survival of the COPI knockdown cohort $(\mathrm{N}=22)$ than the control cohort $(\mathrm{N}=$ 24) (Fig. 4; Mantel-Cox test, $\mathrm{P}<0.001$ ). It has previously been shown that COPI knockdown 
makes the mosquito midgut fragile ${ }^{35}$. Consistently, we observed that the midguts in the COPI knockdown mosquitoes were apt to break and leak during dissection, while the midguts of control mosquitoes were in good shape with intact blood bolus (Fig. 4). A second, replicate experiment also showed a significant reduction in the survival of the COPI knockdown cohort (Mantel-Cox test, $\mathrm{P}<0.01$, Fig. S2).

\section{Cas13a mediated double gene knockdown}

To determine the potential for silencing multiple genes, a cocktail of the Cas 13a construct and crRNAs against the genes Cactus and Caspar or Cactus and COPI was delivered into Ae. aegypti. As expected, the target gene transcripts were reduced effectively by the respective

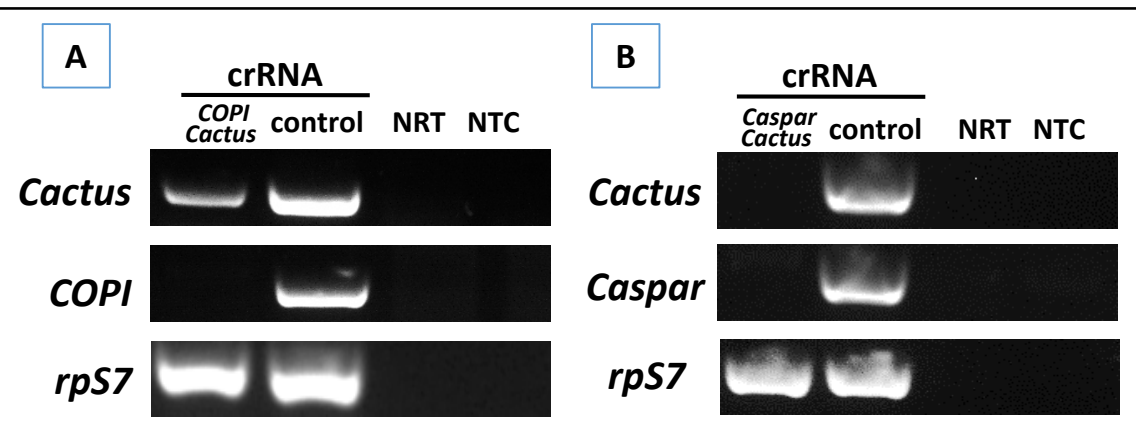

Fig 5. RT-PCR verification of double gene knockdown in Ae. aegypti that received a cocktail of Cas13a construct with (A) Cactus-and Caspar- or (B) Cactus- and COPIcrRNAs. NRT: No RT control; NTC: no template control. The $r p S 7$ was used as a loading control.

treatment (Fig. 5).

Absence of detectable collateral cleavage of non-target RNA 
In bacteria, activated Cas13a displays cleavage activity of non-target RNA ${ }^{10}$. To determine if Cas13a has such collateral activity in mosquitoes, we examined the abundance of arbitrarily selected non-target transcripts in Cas13a/Vg crRNA treated An. gambiae as well as Cas13a/COPICactus crRNA treated Ae. aegypti. As shown in Fig. 6, in An. gambiae, Cas13a/Vg crRNA treatment resulted in $V g$ knockdown, but non-target transcripts $r p S 5, r p S 7$ and GAPDH (glyceraldehyde 3-phosphate dehydrogenase) were not affected by the activated Cas13a. Likewise, in Ae. aegypti, COPI/Cactus crRNA activated Cas13a to co-silence COPI and Cactus, but did not affect non-target transcripts Caspar, rpS7 and $r p S 17$ (Fig. 6). The data indicate that in mosquitoes the Cas13a may not execute collateral cleavage activity on non-target RNA.

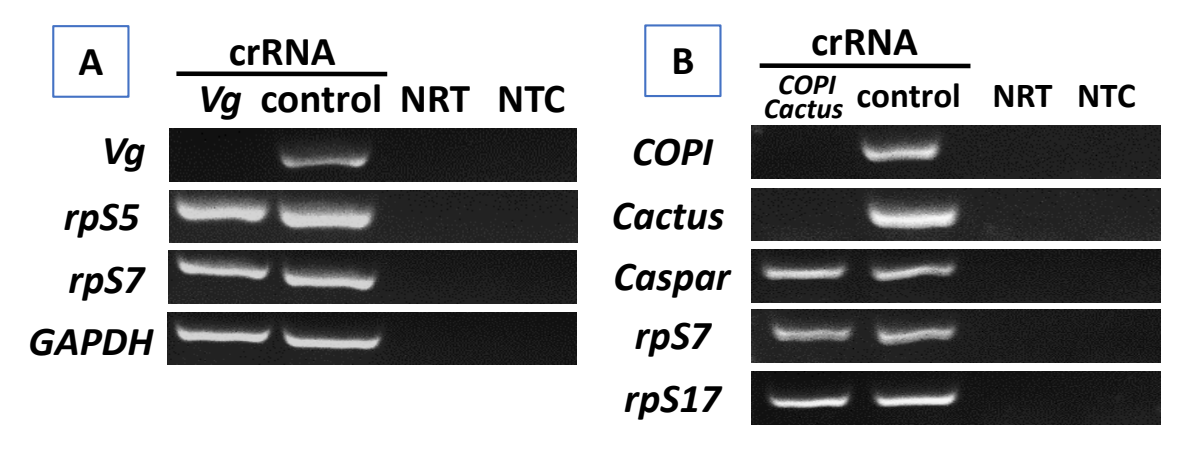

Fig 6. RT-PCR of non-target transcripts. (A) In $V g$ silenced An. gambiae, the abundance of non-target transcripts $r p S 5, r p S 7$, and GAPDH was not affected by Cas $13 \mathrm{a} / \mathrm{Vg}$ crRNA. (B) In COPI and Cactus co-silenced Ae. aegypti, the abundance of non-target transcripts Caspar, rpS7, and rpS17 was not affected by Cas13a/COPI-Cactus crRNA. NRT: No RT control; NTC: no template control.

\section{Discussion}

In this proof of concept study, we demonstrate the effectiveness of CRISPRi mediated by CRISPR-Cas13a/crRNA machinery. The LwaCas13a was derived from L. wadei, and under control of Actin promoter (Fig.1). The construct was delivered into the hemocoel of adult mosquitoes by intrathoracic injection, and Cas 13 a was transcribed constitutively (Fig. 2). Likely, the construct enters into the nucleus where the Cas13a gene is transcribed, and then the mRNA comes into the cytoplasm where the protein is translated. The construct remained active to transcribe Cas13a for at least 10 days post-delivery in Ae. aegypti (Fig. 2), which makes it temporally flexible to administer crRNAs targeting various genes that are expressed at different time points during a mosquito's life span. Target-specific crRNAs can be delivered either with the 
construct together or after the construct delivery as appropriate to the experimental design. The system can silence highly abundant transcripts, as demonstrated by targeting $V g$ transcripts in $A n$. gambiae (Fig. 3) and COPI transcripts in Ae. aegypti (Fig. 4). In both cases, the target genes are induced by blood meal to a high transcriptional abundance. In addition, we have tested the system on the genes Cactus and Caspar in Ae. aegypti (Fig. 5). Taken together, these data conclusively demonstrate that the Cas13a-CRISPRi machinery is functional in An. gambiae and Ae. aegypti mosquitoes we tested in this study. This tool may work well in other mosquito species. We have not tested knockdown effect of the Cas13a system on genes that are mainly expressed in midgut, salivary glands and ovaries, which needs further studies. It would be an appropriate approach to develop a transgenic line expressing Cas13a gene for such studies.

The Cas13a-CRISPRi has certain advantages over the dCas9-CRISPRi for repressing gene expression 16,36. First, the dCas9-CRISPRi machinery acts at DNA level while Cas13a targets mRNA directly. RNA-guided binding of dCas9 to a specific promoter or coding sequence can block transcription. This mode of action is efficient in bacteria, but often is not very efficient in eukaryotic cells ${ }^{16}$. The dCas9 fusion proteins with a repressive domain have been developed for transcriptional repression, such as dCas9-KRAB (Krüppel associated box) in mammalian cells ${ }^{15}$, but it is challenging to develop a fusion dCas9 with universal applicability. In addition, target specific sgRNA selection may be limited by the PAM that is required for the Cas9 activity. On the contrary, no PFS is required for target RNA cleavage by LwaCas13a in eukaryotic cells ${ }^{23}$. The mode of action of Cas13 is simple and programmable, and the efficacy has proven high in mammalian cells ${ }^{11,25}$ and in mosquitoes in the current study. RNAi mediated gene silencing is a very common practice in mosquito gene function studies. Recently, application versions have been developed for mosquito vector control ${ }^{4,5}$. In these application cases, dsRNA is used to trigger RNAi machinery. In dsRNA mediated RNAi, the effective siRNA sequences sometimes are difficult to predict, therefore, large dsRNA fragments are often used to increase chances to generate effective siRNA by Dicer. However, this strategy is accompanied with a higher chance to produce siRNA with off-target potentials ${ }^{37,38}$. In addition, efficacy of dsRNA mediated RNAi varies case by case, and an optimal outcome is often a result of an empirical process ${ }^{39}$.

There is a concern about the potential of collateral cleavage with Cas13a, in which nontarget RNA sequences can be cleaved by Cas13a in bacteria ${ }^{10,11}$. Interestingly, this promiscuous RNA degradation activity was not observed in several studies in mammalian and plant cells ${ }^{11,25,}$ 
26. These data have warranted its safety to be used as an effector to target against RNA viruses that infect humans ${ }^{23}$. However, a Cas13a/crRNA associated collateral cleavage was recently shown in human U87 glioblastoma cells ${ }^{27}$. In the study, exogenous gene GFP or EGFRVIII were overexpressed and targeted by Cas13a/crRNA. In this context, a partial degradation of ribosomal RNA profile was observed, and the abundance of non-target transcripts of GAPDH, HOTHAIR and L3MTL1 was reduced as well ${ }^{27}$. Furthermore, the RNA integrity was compared between the LN229 glioma cell line and HEK293T cells after treatment with the Cas13a/crRNA, the LN229 cells tended to be more sensitive to the collateral effect than the HEK293T cells ${ }^{27}$. In our study, we examined arbitrarily selected non-target transcripts, three in $V g$ silenced An. gambiae and three in COPI and Cactus co-silenced Ae. aegypti; no detectable reduction of these non-target mRNAs was observed (Fig. 6). The data suggest that collateral effect of Cas13a may not be a concern in mosquitoes, although we cannot completely rule out the possibility of collateral cleavage. Additional studies with larger scale examination of non-target RNA would help confirm the absence of non-target cleavage by the Cas13/crRNA system in mosquitoes.

The Cas13a-CRISPRi system holds promise for robust and flexible programming to silence one or more genes simultaneously in mosquitoes, with potential applications in other arthropods.

\section{Acknowledgments}

This research is supported by an Institutional Development Award (IDeA) from the National Institute of General Medical Sciences of the National Institutes of Health under grant number P20GM103451, the National Institutes of Health SC1AI112786 and the National Science Foundation [No. 1633330]. The content is solely the responsibility of the authors.

\section{Author Disclosure Statements}

No competing financial interests exist.

\section{Authorship Confirmation Statement}

JX conceived the idea for the project and devised the study. JX, AK, KAH designed experiments, AK, WY, ASM, AP conducted experiments. AK, JX, KAH wrote manuscript. The authors confirm that all co-authors have reviewed and approved the manuscript. The authors affirm that the paper is original with unpublished findings, not under consideration by any other journals. 


\section{References}

1. Lopez SBG, Guimaraes-Ribeiro V, Rodriguez JVG et al. RNAi-based bioinsecticide for Aedes mosquito control. Sci Rep. 2019;9:4038. DOI: 10.1038/s41598-019-39666-5

2. Whitten MM. Novel RNAi delivery systems in the control of medical and veterinary pests. Curr Opin Insect Sci. 2019;34:1-6. DOI: 10.1016/j.cois.2019.02.001

3. Zhang H, Li HC, Miao XX. Feasibility, limitation and possible solutions of RNAi-based technology for insect pest control. Insect Sci. 2013;20:15-30. DOI: $10.1111 / \mathrm{j} .1744-$ 7917.2012.01513.x

4. Airs PM, Bartholomay LC. RNA Interference for Mosquito and Mosquito-Borne Disease Control. Insects. 2017;8. DOI: 10.3390/insects8010004

5. Balakrishna Pillai A, Nagarajan U, Mitra A et al. RNA interference in mosquito: understanding immune responses, double-stranded RNA delivery systems and potential applications in vector control. Insect Mol Biol. 2017;26:127-139. DOI: 10.1111/imb.12282

6. Chaverra-Rodriguez D, Macias VM, Hughes GL et al. Targeted delivery of CRISPRCas9 ribonucleoprotein into arthropod ovaries for heritable germline gene editing. Nat Commun. 2018;9:3008. DOI: 10.1038/s41467-018-05425-9

7. Li M, Bui M, Yang T et al. Germline Cas9 expression yields highly efficient genome engineering in a major worldwide disease vector, Aedes aegypti. Proc Natl Acad Sci U S A. 2017;114:E10540-E10549. DOI: 10.1073/pnas.1711538114

8. Kistler KE, Vosshall LB, Matthews BJ. Genome engineering with CRISPR-Cas9 in the mosquito Aedes aegypti. Cell Rep. 2015;11:51-60. DOI: 10.1016/j.celrep.2015.03.009

9. Jinek M, Chylinski K, Fonfara I et al. A programmable dual-RNA-guided DNA endonuclease in adaptive bacterial immunity. Science. 2012;337:816-821. DOI:

10.1126/science. 1225829

10. Abudayyeh OO, Gootenberg JS, Konermann S et al. C2c2 is a single-component programmable RNA-guided RNA-targeting CRISPR effector. Science. 2016;353:aaf5573. DOI: 10.1126/science.aaf5573

11. Abudayyeh OO, Gootenberg JS, Essletzbichler P et al. RNA targeting with CRISPRCas13. Nature. 2017;550:280-284. DOI: 10.1038/nature24049

12. Makarova KS, Wolf YI, Alkhnbashi OS et al. An updated evolutionary classification of CRISPR-Cas systems. Nat Rev Microbiol. 2015;13:722-736. DOI: 10.1038/nrmicro3569

13. Zhang F, Wen Y, Guo X. CRISPR/Cas9 for genome editing: progress, implications and challenges. Hum Mol Genet. 2014;23:R40-46. DOI: 10.1093/hmg/ddu125

14. Doudna JA, Charpentier E. Genome editing. The new frontier of genome engineering with CRISPR-Cas9. Science. 2014;346:1258096. DOI: 10.1126/science.1258096

15. Gilbert LA, Larson MH, Morsut L et al. CRISPR-mediated modular RNA-guided regulation of transcription in eukaryotes. Cell. 2013;154:442-451. DOI:

10.1016/j.cell.2013.06.044

16. Qi LS, Larson MH, Gilbert LA et al. Repurposing CRISPR as an RNA-guided platform for sequence-specific control of gene expression. Cell. 2013;152:1173-1183. DOI:

10.1016/j.cell.2013.02.022

17. Bikard D, Jiang W, Samai $P$ et al. Programmable repression and activation of bacterial gene expression using an engineered CRISPR-Cas system. Nucleic Acids Res. 2013;41:74297437. DOI: $10.1093 /$ nar/gkt520 
18. Shmakov S, Abudayyeh OO, Makarova KS et al. Discovery and Functional Characterization of Diverse Class 2 CRISPR-Cas Systems. Mol Cell. 2015;60:385-397. DOI: 10.1016/j.molcel.2015.10.008

19. Zetsche B, Gootenberg JS, Abudayyeh OO et al. Cpf1 is a single RNA-guided endonuclease of a class 2 CRISPR-Cas system. Cell. 2015;163:759-771. DOI: 10.1016/j.cell.2015.09.038

20. Aman R, Ali Z, Butt $\mathrm{H}$ et al. RNA virus interference via CRISPR/Cas13a system in plants. Genome Biol. 2018;19:1. DOI: 10.1186/s13059-017-1381-1

21. Ali Z, Mahas A, Mahfouz M. CRISPR/Cas13 as a Tool for RNA Interference. Trends Plant Sci. 2018;23:374-378. DOI: 10.1016/j.tplants.2018.03.003

22. Terns MP. CRISPR-Based Technologies: Impact of RNA-Targeting Systems. Mol Cell. 2018;72:404-412. DOI: 10.1016/j.molcel.2018.09.018

23. Freije CA, Myhrvold C, Boehm CK et al. Programmable Inhibition and Detection of RNA Viruses Using Cas13. Mol Cell. 2019. DOI: 10.1016/j.molcel.2019.09.013

24. Liu L, Li X, Ma J et al. The Molecular Architecture for RNA-Guided RNA Cleavage by Cas13a. Cell. 2017;170:714-726 e710. DOI: 10.1016/j.cell.2017.06.050

25. Cox DBT, Gootenberg JS, Abudayyeh OO et al. RNA editing with CRISPR-Cas13. Science. 2017;358:1019-1027. DOI: 10.1126/science.aaq0180

26. Konermann S, Lotfy P, Brideau NJ et al. Transcriptome Engineering with RNATargeting Type VI-D CRISPR Effectors. Cell. 2018;173:665-676 e614. DOI: 10.1016/j.cell.2018.02.033

27. Wang Q, Liu X, Zhou J et al. The CRISPR-Cas13a Gene-Editing System Induces Collateral Cleavage of RNA in Glioma Cells. Adv Sci (Weinh). 2019;6:1901299. DOI: 10.1002/advs.201901299

28. Gootenberg JS, Abudayyeh OO, Lee JW et al. Nucleic acid detection with CRISPRCas13a/C2c2. Science. 2017;356:438-442. DOI: 10.1126/science.aam9321

29. Pei Y, Lu M. Programmable RNA manipulation in living cells. Cell Mol Life Sci. 2019. DOI: $10.1007 / \mathrm{s} 00018-019-03252-9$

30. Zhao X, Liu L, Lang J et al. A CRISPR-Cas13a system for efficient and specific therapeutic targeting of mutant KRAS for pancreatic cancer treatment. Cancer Lett. 2018;431:171-181. DOI: 10.1016/j.canlet.2018.05.042

31. Kandel Y, Vulcan J, Rodriguez SD et al. Widespread insecticide resistance in Aedes aegypti L. from New Mexico, U.S.A. PLoS One. 2019;14:e0212693. DOI:

10.1371/journal.pone.0212693

32. Wang Y, Gilbreath TM, 3rd, Kukutla P et al. Dynamic gut microbiome across life history of the malaria mosquito Anopheles gambiae in Kenya. PLoS One. 2011;6:e24767. DOI: 10.1371/journal.pone.0024767

33. Bassett AR, Tibbit C, Ponting CP et al. Mutagenesis and homologous recombination in Drosophila cell lines using CRISPR/Cas9. Biol Open. 2014;3:42-49. DOI: 10.1242/bio.20137120 34. CLEMENTSM AN, BOOCOCK R. Ovarian development in mosquitoes: Stages of growth and arrest, and follicular resorption. Physiological Entomology. 2008;9:1-8.

35. Isoe J, Collins J, Badgandi $\mathrm{H}$ et al. Defects in coatomer protein I (COPI) transport cause blood feeding-induced mortality in Yellow Fever mosquitoes. Proc Natl Acad Sci U S A. 2011;108:E211-217. DOI: 10.1073/pnas.1102637108

36. Xu X, Qi LS. A CRISPR-dCas Toolbox for Genetic Engineering and Synthetic Biology. J Mol Biol. 2019;431:34-47. DOI: 10.1016/j.jmb.2018.06.037 
37. Naito Y, Yamada T, Matsumiya T et al. dsCheck: highly sensitive off-target search software for double-stranded RNA-mediated RNA interference. Nucleic Acids Res.

2005;33:W589-591. DOI: 10.1093/nar/gki419

38. Jackson AL, Bartz SR, Schelter J et al. Expression profiling reveals off-target gene regulation by RNAi. Nat Biotechnol. 2003;21:635-637. DOI: 10.1038/nbt831

39. Scott JG, Michel K, Bartholomay LC et al. Towards the elements of successful insect RNAi. J Insect Physiol. 2013;59:1212-1221. DOI: 10.1016/j.jinsphys.2013.08.014

Table S1. Primer sets used in the study

\begin{tabular}{llc}
\hline Primer & Sequence & Amplicon Size (bp) \\
\hline Aa_Cactus_F & AGACAGCCGCACCTTCGATTCC & 232 \\
Aa_Cactus_R & CGCTTCGGTAGCCTCGTGGATC & 270 \\
\hline Aa_Caspar_F & GAATCCGAGCGAGCCGATGC & 252 \\
Aa_Caspar_R & CGTAGTCCAGCGTTGTGAGGTC & \\
\hline Aa_rpS7_F & AAGGTCGACACCTTCACGTC & 240 \\
Aa_rpS7_R & TCTTGTCCCTCCGTTTGGTG & 256 \\
\hline Aa_rpS17_F & CGTGAGCGCAGAGACAACTA & \multirow{2}{*}{442} \\
Aa_rpS17_R & ACCATGGATGTTCCGGTGTG & \\
\hline Aa_COPI_F & GACGTTGCGCATATCAGACG & 250 \\
Aa_COPI_R & CAGCATTCTCAGAGGGCCAA & \\
\hline Cas13a_F & TCCGCCAACAAGGAAGAGAC & 211 \\
Cas13a_R & CCGATGGCCTTCTCGTACTC & \\
\hline Ag_Vg_F & ACTTCTTCCAGGGCAAGCAC & \\
Ag_Vg_R & CACAGCGCAAGATGGATGGT & \\
\hline Ag_rpS7_F & GCGTGAGGTCGAGTTCAACA & \\
Ag_rpS7_R & GGGAACGCGGTCTCTTCTG & \\
\hline Ag_rpS5_F & CCATGTCACGTCTCGTCACT & \\
Ag_rpS5_R & CGAAAACCATCCACACACAC & \\
\hline
\end{tabular}




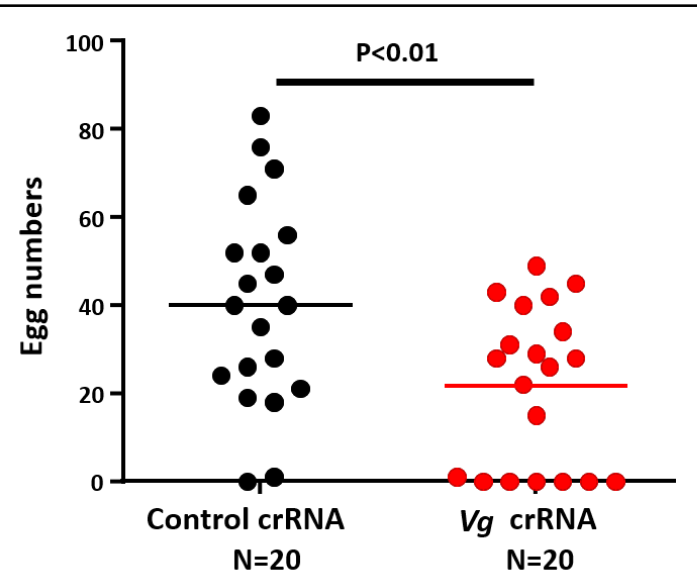

Figure S1. The Cas $13 \mathrm{a} / \mathrm{Vg}$ crRNA treatment significantly reduced egg production in An. gambiae. Mann-Whitney test, $\mathrm{P}<0.01$.

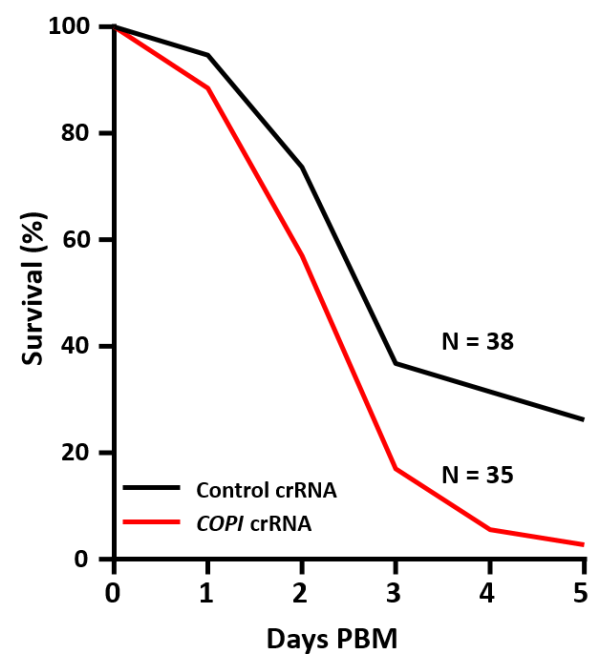

Figure S2. The Cas13a/COPI-crRNA treatment resulted in a significant higher mortality post a blood meal. Mantel Cox test, $\mathrm{P}<0.01$. 